\title{
Election of President \\ Notice to Fellows and Members
}

Fellows and Members are reminded of their rights under the Bye-laws and Regulations, as follows:

\section{Bye-law XI}

The President shall be elected annually from amongst the Fellows.

\section{Regulation XI}

(1) As soon as may be practicable after the first day of January in any year the Council shall hold a nomination meeting and shall... nominate not less than one candidate and not more than three candidates...
(2) Between the first day of January and the date which is four clear weeks after the nomination meeting of the Council, written nominations, accompanied by the nominees' written consent to stand for election, may be lodged with the Registrar, provided that each such nomination is supported in writing by not less than twelve Members of the College who are not members of the Council.

(3) An election by ballot shall be held in accordance with the provisions of the Regulations.

The nominating meeting of the Council will be held on 18 January 1983, and the last date for receiving nominations under (2) above will therefore be 16 February 1983.

\section{Elections to the Fellowship, 1981-2}

The following Members have registered as Fellows of the College following their election.

\section{1}

B. Alapin, D. Abrahamson, G. W. Ashcrof, B. R. Ballinger, E. D. Barlow, B. M. Barraclough, C. Berry, J. A. Birtchnell, A. A. Black, J. M. Black, M. R. Bond, J. A. Bonn, C. M. Brennan, I. G. Bronks, B. V. Burke, J. J. Byng-Hall, J. Caldbeck-Meenan, H. C. Cameron, J. A. Cameron, J. Candy, D. M. Carter, E. L. Cay, I. D. Chisholm, A. K. M. D. Chowdhury, J. Connolly, A. I. Cooklin, N. Murray Cox, M. W. Coxon, T. J. Crow, R. L. Cundall, J. Currah, C. Davies, Margaret E. B. Davies, M. H. Davies, D. H. Dick, J. H. Dowson, D. L. F. Dunleavy, Deepali Dutta, W. M. Easson, A. C. Fairburn, J. Farr, E. A. Frommer, A. Galea, H. K. Gallagher, R. Gardner, M. J. Gay, A. I. M. Glen, C. Godber, A. S. R. Goonetilleke, L. K. Hemsi, Doris Hollander, F. A. S. Jensen, D. J. Jolley, M. P. JoystonBechal, P. W. Kershaw, D. F. Klein, M. S. Lipsedge, G. A. Low-Beer, M. D. Lynch, R. MacDonald, A. S. MacVicar, R. V. Magnus, T. M. Maguire, A. A. Marinho, D. H. Marjot, H. M. McBryde, J. L. McClure, J. McDonald, C. P. McEvedy, D. L. M. McNeill, P. I. Melia, D. W. Millard, D. H. Morgan, Y Myers, N. P. V. Nair, A. R. Nicol, F. D. Pascoe, S. M. W. Pittock, D. A. Player, I. G. Pryce, V.
Ramachandran, A. V. N. Rao, Naomi S. Richman, H. K. Rose, B. M. Saran, B. G. Scally, J. C. Scott, D. Shaffer, J. G. Simeon, A. N. Singh, S. D. Soni, M. S. Spelman, W. B. Spry, W. J. Stanley, J. Steiner, G. Stores, M. Subramaniam, P. E. Sylvester, E. Szabadi, L. Tarlo, R. L. Tyson, E. L. Udwin, A. Verghese, J. D. Waldman, J. Warburton, J. L. F. Warnants, M. C. Way, P. D. Wickenden, M. E. F. Woollaston, E. G. Wooster, A. R. Worthers, S. C. B. Yorke, M. E. YorkMoore.

\section{2}

P. F. J. Aaronricks, F. Arroyave-Portela, G. E. Berrios, G. N. Bianchi, R. H. Boardman, P. Boyd, A. M. Bradfield, J. Brunning, A. S. Carey, J. R. Cornwell, B. Davies, R. J. Davies, P. B. de Mare, W. G. Dewhurst, R. J. Draper, B. W. Durrant, J. C. Ebie, D. H. Ezzat, A. R. Foster, O. S. Frank, M. S. A. Gawad, S. Gershon, A. B. Goorney, J. R. Hamilton, E. A. Harvey-Smith, C. H. Howard, B. K. Jha, J. M. Kellett, W. B. Knapman, E. S. Lamont, L. I. Liebling, N. C. Low, D. M. MacGregor, S. A. Montgomery, M. H. Mubbashar, G. Mustafa, W. A. Norris, J. R. Robinson, O. H. Shaheen, S. J. Shamsie, H. R. Simons, A. Smith, C. G. Smith, D. A. Spencer, K. Standage, J. Templeton, D. A. Toms, H. G. Tough, M. R. Trimble, D. P. Wheatley, J. A. Whitehead, J. S. Whiteley, W. A. Zaleski, H. Zeitlin. 